\title{
ON THE SOLUTION TO A CLASS OF STRONGLY SINGULAR LINEAR INTEGRAL EQUATIONS
}

\author{
BY \\ A. K. GAUTESEN \\ Iowa State University, Ames, Iowa
}

\begin{abstract}
A strongly singular linear integral equation containing a small positive parameter $\delta$ is considered. This equation is transformed into a Fredholm integral equation of the second kind with a continuous kernel. The rate of convergence of the Neumann series for this integral equation is shown to be $o\left(\delta^{2}\right)$. An example from fracture mechanics is considered in detail.
\end{abstract}

1. Introduction. We consider the following integral equation

$$
\begin{gathered}
u(x)+\delta L_{x} L_{x}^{*} \int_{0}^{1} m(x-y) u(y) d y=f_{0}(x), \quad 0<x<1, \\
u(0)=u(1)=0,
\end{gathered}
$$

where

$$
L_{x}=d / d x-i \alpha, \quad L_{x}^{*}=-d / d x-i \alpha,
$$

$\delta$ is a small positive parameter, and $\alpha$ is a constant with $\operatorname{Im}\{\alpha\} \geq 0$. The kernel $m(x)$ is assumed to have a singularity at $x=0$. Thus the integral equation is hypersingular. The linearity of (1.1) plays an integral part in the method of solution developed herein.

A recent theoretical investigation by Nemat-Nasser and Hori [3] of the bridging of cracks in brittle materials through fiber reinforcement has provided a formulation of the problem as an integral equation. For the special case of a crack normal to the fibers with constant bridging stiffness, the integral equation takes the form (1.1) with $\alpha=0$ and $m(x)=-\pi^{-1} \log |x|$. Olmstead and Gautesen [4], Hori and Nemat-Nasser [2], and Willis and Nemat-Nasser [5] have considered this example asymptotically for small $\delta$. In the first two papers the authors give the leading order term in the asymptotic expansion of the solution $u$ without any indication of how to obtain higher-order terms. Only in the latter work is a systematic method for obtaining the higher terms given. They use the method of inner and outer expansions to obtain the solution to $o\left(\delta^{2}\right)$. By this method, however, proceeding to higher-order terms is laborious. In addition, when their asymptotic approximation for $u$ to any 
order in $\delta$ is substituted into (1.1a), singularities occur at $x=0$ and 1. Our method does not have this difficulty.

The main result of this work is to show that under suitable assumptions about the kernel $k(x), u(x)$ satisfies the Fredholm integral equation of the second kind

$$
u(x)+\delta \int_{0}^{1} m_{1}(x, y) u(y) d y=F_{0}(x), \quad 0<x<1,
$$

where $m_{1}(x, y)$ and $F_{0}(x)$ are given by (2.44) and (2.47) below. These functions are continuous functions of their variables and vanish when $x=0,1$. For $\delta$ small, $m_{1}(x, y) \sim o(\delta)$ uniformly in $x$ and $y$. Thus, we immediately have the approximation $u(x) \sim F_{0}(x)+o\left(\delta^{2}\right)$. For the aforementioned example the error in this approximation is $O\left(\delta^{3} \log \delta\right)$.

We make the following assumptions about the Fourier transform $\hat{m}(\xi)$ of the kernel $m(x)$, which is defined by

$$
\hat{m}(\xi)=\int_{-\infty}^{\infty} e^{i \xi x} m(x) d x
$$

First we assume that

$$
\hat{m}(\xi)=\hat{m}(-\xi) .
$$

Then on the region in the complex $\xi$-plane, which is the union of $|\operatorname{Im}\{\xi\}|<2 \tau_{i}$ and $|\operatorname{Re}\{\xi\}|>\frac{1}{2} \sigma_{r}$, we assume that

$$
\begin{gathered}
\hat{m}(\xi) \text { is analytic; } \\
\hat{m}(\xi) \sim O\left(|\xi|^{-1+\gamma}\right), \quad \text { as } \xi \rightarrow \infty, 0 \leq \gamma<1 ; \\
1+\delta\left(\xi^{2}-\alpha^{2}\right) \hat{m}(\xi) \neq 0 .
\end{gathered}
$$

The first assumption (1.5) implies that the kernel is symmetric,

$$
m(x)=m(-x) \text {. }
$$

The kernel $m(x)$ is given by

$$
m(x)=\frac{1}{2 \pi} \int_{-\infty}^{\infty} e^{-i \xi x} \hat{m}(\xi) d \xi .
$$

For $x>0$, assumptions (1.6) and (1.7) allow us to deform the $\xi$-contour in (1.10) to the contour $C_{\xi}$ where for $\xi=\sigma+i \tau$,

$$
C_{\xi}=\left\{\sigma=-\sigma_{r}, \tau \leq-\tau_{i}\right\} \cup\left\{|\sigma|<\sigma_{r}, \tau=-\tau_{i}\right\} \cup\left\{\sigma=\sigma_{r}, \quad \tau \leq-\tau_{i}\right\} .
$$

Thus

$$
m(x)=\frac{1}{2 \pi} \int_{C_{\xi}} e^{-i \xi x} \hat{m}(\xi) d \xi, \quad x>0 .
$$

From (1.12) it follows that $m(x)$ and all its derivatives are continuous for $x>0$. At $x=0$ the kernel $m(x)$ is singular, which follows from (1.10) and (1.7).

We need a Wiener-Hopf factorization of

$$
\hat{n}(\xi)=c_{0}\left[1+\delta\left(\xi^{2}-\alpha^{2}\right) \hat{m}(\xi)\right]^{-1},
$$


where

By (1.5) we can write

$$
c_{0}=1-\delta \alpha^{2} \hat{m}(0)
$$

$$
\hat{n}(\xi)=\hat{b}(\xi) \hat{b}(-\xi),
$$

where $\hat{b}(\xi)$ is analytic for $\operatorname{Im}\{\xi\}>-\tau_{i}$. An explicit expression for $\hat{b}(z)$ follows from assumptions (1.6) $-(1.8)$ as

$$
\begin{aligned}
\log \hat{b}(z) & =\frac{1}{2 \pi i} \int_{-\infty-i \tau_{i}}^{\infty-i \tau_{i}} \log \hat{n}(\xi)\left[\frac{1}{\xi-z}-\frac{1}{\xi}\right] d \xi \\
& =\frac{i}{2 \pi} \int_{C_{\xi}} \log \left[1+\delta\left(\xi^{2}-\alpha^{2}\right) \hat{m}(\xi)\right]\left(\frac{1}{\xi-z}-\frac{1}{\xi}\right) d \xi
\end{aligned}
$$

Note that

$$
\hat{b}(0)=1
$$

and that

$$
\lim _{\delta \rightarrow 0} \hat{b}(\xi)=1 .
$$

For small $\delta$ we need an asymptotic expression for $b(x)$, the inverse Fourier transform of $\hat{b}(\xi)$. We can write

$$
\begin{aligned}
b(x) & =\frac{1}{2 \pi} \int_{-\infty}^{\infty} \exp [-i x z+\log \hat{b}(z)] d z \\
& =\frac{1}{2 \pi} \int_{C_{z}} \exp [-i x z+\log \hat{b}(z)] d z, \quad x>0,
\end{aligned}
$$

where the contour $C_{z}$ lies just outside of the contour $C_{\xi}$ defined by (1.11). For $|z|$ large, the integrand in (1.19) has exponential decay. Thus for small $\delta$ and $x>0$, we write

$$
b(x) \sim \frac{1}{2 \pi} \int_{C_{z}} e^{-i x z}\{1+\log \hat{b}(z)+\cdots\} d z .
$$

The integral of the first term is zero and as a function of $z, \log \hat{b}(z)$ has a pole at $z=\xi$. Thus (1.20) becomes

$$
\begin{aligned}
b(x) & \sim-\frac{1}{2 \pi} \int_{C_{\xi}} e^{-i x \xi} \log \left[1+\delta\left(\xi^{2}-\alpha^{2}\right) \hat{m}(\xi)\right] d \xi \\
& \sim-\frac{\delta}{2 \pi} \int_{C_{\xi}} e^{-i x \xi}\left(\xi^{2}-\alpha^{2}\right) \hat{m}(\xi) d \xi
\end{aligned}
$$

The last integral follows from (1.12). Thus we assume we can write

$$
b(x) \sim \delta b_{1}(x)+o(\delta), \quad x>0,
$$

where

$$
b_{1}(x)=m^{\prime \prime}(x)+\alpha^{2} m(x)
$$

and $m^{\prime \prime}(x)$ denotes the second derivative.

In the next section we derive our main result (1.3). In the last section we show that $m_{1}(x, y) \sim o(\delta)$ and consider in detail the example from fracture mechanics. Also, we briefly indicate the changes required when the first term $u(x)$ in (1.1a) is replaced by $q(x) u(x)$. 
2. Integral identities. We can also write the integral equation (1.1) as

$$
\begin{aligned}
K u=L_{x} L_{x}^{*} \int_{0}^{1} k(x-y) u(y) d y & =f(x), \quad 0<x<1, \\
u(1)=u(0) & =0,
\end{aligned}
$$

where

$$
\begin{aligned}
& k(x)=-\left(2 i \alpha c_{0}\right)^{-1} \exp [i \alpha|x|]+\delta m_{0}(x), \\
& m_{0}(x)=m(x) / c_{0}, \quad f(x)=f_{0}(x) / c_{0},
\end{aligned}
$$

and $c_{0}$ is given by (1.14), and without loss of generality, we can assume that

$$
f(x)=\varepsilon f(1-x), \quad \varepsilon=1 \text { or }-1 .
$$

If $f$ does not satisfy (2.4), we write $f(x)=f_{1}(x)+f_{2}(x)$ where $f_{1}(x)=\frac{1}{2}(f(x)+$ $f(1-x))$ and $f_{2}(x)=\frac{1}{2}(f(x)-f(1-x))$. Then $f_{1}$ and $f_{2}$ satisfy (2.4). Since $K$ is linear, we can write $u=u_{1}+u_{2}$ where $K u_{i}=f_{i}$. As a consequence of (2.4), we have

$$
u(x)=\varepsilon u(1-x) .
$$

We show below that (2.1) can also be expressed as

$$
u(x)=F(x)-U(x)-\varepsilon U\left(x_{0}\right),
$$

where

$$
\begin{gathered}
x_{0}=(1-x) ; \\
F(x)=F_{1}(x)+\varepsilon F_{1}\left(x_{0}\right)-N f ; \\
N f=\int_{0}^{1} n(x-y) f(y) d y ; \\
n(x-y)=\int_{\max (x, y)}^{\infty} b(s-x) b(s-y) d s=\int_{-\infty}^{\min (x, y)} b(x-s) b(y-s) d s ; \\
F_{1}(x)=\int_{0}^{x} b(x-s) \int_{s}^{1} b(y-s) f(y) d y d s ; \\
U(x)=\int_{0}^{\infty} b(x+s) U_{1}(s) d s ; \\
U_{1}(s)=\delta \int_{0}^{1} \int_{0}^{\infty} b(1+s+t) L_{t} L_{t}^{*} m_{0}(1-y+t) u(y) d t d y ;
\end{gathered}
$$

and $b(x)$ is given by (1.19). We shall now write (2.6) in a different form. By repeated integration by parts we can also express $U_{1}(s)$ as

$$
\begin{aligned}
U_{1}(s)= & \delta \int_{0}^{1} \int_{0}^{\infty} m_{0}(1-y+t) L_{t} L_{t}^{*} b(1+s+t) u(y) d t d y \\
& -b^{\prime}(1+s) C_{1}-b(1+s) C_{2}
\end{aligned}
$$


where the prime denotes the derivative and

$$
\begin{aligned}
& C_{1}=\delta \int_{0}^{1} m_{0}(1-y) u(y) d y, \\
& C_{2}=-\delta \int_{0}^{1} m_{0}(1-y) u^{\prime}(y) d y .
\end{aligned}
$$

Then

$$
U(x)+\varepsilon U\left(x_{0}\right)=V(x)-V_{1}(x) C_{1}-V_{2}(x) C_{2},
$$

where

$$
\begin{gathered}
V(x)=\delta \int_{0}^{1} \int_{0}^{\infty}\left\{m_{0}(1-y+t) L_{t} L_{t}^{*} g(x, 1+t)\right. \\
\left.+m_{0}(y+t) L_{t} L_{t}^{*} g\left(x_{0}, 1+t\right)\right\} u(y) d t d y \\
V_{1}(x)=g_{t}(x, 1)+\varepsilon g_{t}\left(x_{0}, 1\right) \\
V_{2}(x)=g(x, 1)+\varepsilon g\left(x_{0}, 1\right) \\
g(x, t)=\int_{0}^{\infty} b(x+s) b(s+t) d s
\end{gathered}
$$

and $g_{t}(x, t)$ denotes the partial derivative of $g(x, t)$ with respect to $t$. Also, it can be shown that

$$
\begin{aligned}
F(x)= & \int_{0}^{x} \int_{0}^{x_{0}} b(s) b(y) f(x+y-s) d y d s \\
& -\int_{x_{0}}^{1} g(x, y) f(y) d y-\int_{0}^{x_{0}} g\left(x_{0}, 1-y\right) f(y) d y .
\end{aligned}
$$

Thus (2.6) becomes

$$
u(x)=F(x)-V(x)+C_{1} V_{1}(x)+C_{2} V_{2}(x) .
$$

We shall now obtain an alternate expression for the constants $C_{1}$ and $C_{2}$. Since $u(1)=0,(2.23)$ yields

$$
0=F(1)-V(1)+C_{1} V_{1}(1)+C_{2} V_{2}(1) .
$$

We remark that $(2.24)$ also assures that $u(0)=0$. Near $x=0$,

$$
u(x) \sim C_{3} B(x)+C_{4} x+o(x),
$$

where

$$
\begin{gathered}
B(x)=\int_{0}^{x} b(t) d t \\
C_{3}=\int_{0}^{1} b(y) f(y) d y-b^{\prime}(1) C_{1}-b(1) C_{2}+C_{5} ; \\
C_{4}=\int_{0}^{1} g_{x}(1, y) f(y) d y-\left(g_{t t}(0,1)+\varepsilon g_{x t}(1,1)\right) C_{1} \\
-\left(g_{t}(0,1)+\varepsilon g_{x}(1,1)\right) C_{2}+C_{6} ;
\end{gathered}
$$




$$
\begin{gathered}
C_{5}=\delta \int_{0}^{1} \int_{0}^{\infty} m_{0}(1-y+t) L_{t} L_{t}^{*} b(1+t) u(y) d t d y \\
C_{6}=\delta \int_{0}^{1} \int_{0}^{\infty}\left\{\begin{array}{r}
m_{0}(1-y+t) L_{t} L_{t}^{*} g_{t}(0,1+t) \\
\left.+m_{0}(y+t) L_{t} L_{t}^{*} g_{x}(1,1+t)\right\} u(y) d t d y .
\end{array}\right.
\end{gathered}
$$

To establish (2.25) it is sufficient to show that

$$
\lim _{x \rightarrow 0}\left(u^{\prime}(x)-C_{3} b(x)\right)=C_{4},
$$

where $u^{\prime}(x)$ denotes the derivative of $u(x)$. This relation follows by differentiating (2.23) and using the identity

$$
g_{x}(x, t)=-b(x) b(t)-g_{t}(x, t),
$$

where the subscripts denote the partial derivative. Substituting $u(x)=B(x)$ into (2.1a) and noting the identity

$$
L_{x} L_{x}^{*} \int_{0}^{\infty} k(x-y) B(y) d y=1, \quad x>0,
$$

we find that the resulting function of $x$ is regular near $x=0$. However, when $u=x,(2.1 \mathrm{a})$ becomes

$$
K u \sim-k(x) \text { as } x \rightarrow 0,
$$

which is singular at $x=0$. Thus by (2.25) it follows that

$$
C_{4}=0 \text {. }
$$

Instead of solving (2.24) and (2.28) for $C_{1}$ and $C_{2}$, we find it convenient to define

$$
C_{1} V_{1}(x)+C_{2} V_{2}(x)=D_{1} W_{1}(x)+D_{2} W_{2}(x),
$$

where

$$
\begin{aligned}
& 2 V_{1}(1) V_{2}(1) W_{1}(x) \\
& =V_{2}(1) V_{1}(x)+V_{1}(1) V_{2}(x)-\left[V_{1}(1) g_{1}+V_{2}(1) g_{2}\right] W_{2}(x) \\
& W_{2}(x)=\left[V_{2}(1) V_{1}(x)-V_{1}(1) V_{2}(x)\right] /\left[V_{2}(1) g_{2}-V_{1}(1) g_{1}\right] \\
& g_{1}=g_{t}(0,1)+\varepsilon g_{x}(1,1) \\
& g_{2}=g_{t t}(0,1)+\varepsilon g_{x t}(1,1) .
\end{aligned}
$$

Then (2.24) and (2.28), respectively, become

$$
\begin{gathered}
D_{1}=V(1)-F(1), \\
D_{2}=C_{6}+\int_{0}^{1} g_{x}(1, y) f(y) d y .
\end{gathered}
$$

Finally, upon substitution from (2.36), (2.41), (2.42), and (2.18), we find that (2.23) becomes

$$
u(x)+\delta \int_{0}^{1} m_{1}(x, y) u(y) d y=F_{0}(x), \quad 0<x<1,
$$


where

$$
\begin{gathered}
m_{1}(x, y)=m_{2}(x, y)-m_{2}(1, y) W_{1}(x)-m_{3}(y) W_{2}(x) \\
m_{2}(x, y)=\int_{0}^{\infty}\left\{m_{0}(1-y+t) L_{t} L_{t}^{*} g(x, 1+t)\right. \\
\left.\quad+m_{0}(y+t) L_{t} L_{t}^{*} g\left(x_{0}, 1+t\right)\right\} d t \\
m_{3}(y)=\int_{0}^{\infty}\left\{m_{0}(1-y+t) L_{t} L_{t}^{*} g_{t}(0,1+t)\right. \\
\left.+m_{0}(y+t) L_{t} L_{t}^{*} g_{x}(1,1+t)\right\} d t \\
F_{0}(x)=F(x)+\int_{0}^{1} g(1, y) f(y) d y W_{1}(x)+\int_{0}^{1} g_{x}(1, y) f(y) d y W_{2}(x)
\end{gathered}
$$

Integral equation (2.43) is a Fredholm integral equation of the second kind whose kernel $m_{1}(x, y)$ is a continuous function of $x$ and $y$. It has the properties that $m_{1}(0, y)=m_{1}(1, y)=0$ and that

$$
\begin{gathered}
m_{1}(x, y) \sim \text { const } B(x)+o(x) \quad \text { as } x \rightarrow 0, \\
m_{1}(x, y) \sim \text { const } B(1-x)+o(1-x) \text { as } x \rightarrow 1
\end{gathered}
$$

uniformly in $y$. Similarly, $F_{0}(x)$ is continuous, vanishes at $x=0$, and satisfies $(2.48)$ when $m_{1}(x, y)$ is replaced by $F_{0}(x)$. If the Neumann series for $(2.43)$ converges, then we can approximate $u$ by a finite number of terms in this series. When this approximation is substituted into the original integral equation $(1.1 \mathrm{a})$, the resulting function of $x$ is continuous.

We have yet to establish (2.6). To this end, we first show that

$$
u(x)=N f+U_{2}\left(x_{0}\right)+\varepsilon U_{2}(x), \quad 0<x<1,
$$

where

$$
U_{2}(x)=\delta \int_{0}^{1} \int_{0}^{\infty} n(x+t) L_{t} L_{t}^{*} m_{0}(1-y+t) u(y) d t d y
$$

and then show that

$$
u(x)=F_{1}(x)+U_{2}\left(x_{0}\right)-U(x) .
$$

Subtracting (2.49) and (2.51) gives

$$
U_{2}(x)=\varepsilon\left(F_{1}(x)-N f-U(x)\right) .
$$

Using (2.52) to eliminate $U_{2}\left(x_{0}\right)$ in $(2.51)$ yields $(2.6)$ upon noting that $(N f)(x)=$ $\varepsilon(N f)\left(x_{0}\right)$.

To establish (2.49) we define $u(x)=0$ and $f(x)=0$ for $x \notin(0,1)$ and define a function $U_{3}(x)$ by $U_{3}(x)=0,0<x<1$ and

$$
U_{3}(x)=L_{x} L_{x}^{*} \int_{0}^{1} k(x-y) u(y) d y=\delta L_{x} L_{x}^{*} \int_{0}^{1} m_{0}(x-y) u(y) d y, \quad x \notin(0,1) .
$$

Then (2.1) can be expressed as

$$
L_{x} L_{x}^{*} \int_{-\infty}^{\infty} k(x-y) u(y) d y=f(x)+U_{3}(x), \quad-\infty<x<\infty .
$$


The inverse of the operator on the left side of (2.54) is the operator $\int_{-\infty}^{\infty} d z n(z-x)$. Thus (2.54) can also be expressed as

$$
u(x)=N f+\int_{1}^{\infty} n(s-x) U_{3}(s) d s+\int_{0}^{\infty} n(x+s) U_{3}(-s) d s, \quad 0<x<1 .
$$

In the first integral we let $s=1+t$ to find that it is given by $U_{2}\left(x_{0}\right)$. From (2.53) it follows that $U_{3}(-x)=\varepsilon U_{3}(1+x)$. The last integral, then, is $\varepsilon U_{2}(x)$. We have thus proved (2.49).

To establish (2.51), we shall use an identity from Gautesen [1]. His equation (1.2) with $\lambda=1$ is identical to our equation (2.1). Thus, we can use his identity (2.28), which is

$$
L_{s} \int_{0}^{s} a(s-y) u(y) d y=\int_{s}^{1} b(y-s) f(y) d y+U_{1}(-s), \quad 0<s<1,
$$

where $U_{1}$ is defined by (2.13). The Fourier transform of $a(x)$ is

$$
\hat{a}(\xi)=i /[\hat{b}(\xi)(\xi+\alpha)] .
$$

It has the property that $\hat{a}(\xi) \hat{a}(-\xi)=\hat{k}(\xi)$, where $\hat{k}(\xi)$ is the Fourier transform of the kernel $k(x)$ in (2.1). Operating on (2.56) with $\int_{0}^{x} d s b(x-s)$ yields

$$
u(x)=F_{1}(x)+\int_{-\infty}^{x} b(x-s) U_{1}(-s) d s-\int_{-\infty}^{0} b(x-s) U_{1}(-s) d s
$$

where $F_{1}(x)$ is given by (2.11). Letting $s=-t$ in the last integral in (2.58) we find by $(2.12)$ that it is $U(x)$. Next we substitute for $U_{1}(-s)$ from (2.13) in the first integral in (2.58) and use (2.10) to perform the $s$ integration. The result is that this integral is $U_{2}\left(x_{0}\right)$, where $U_{2}(x)$ is given by (2.50). Thus we have proved (2.51).

3. Asymptotics and example. In this section we consider the integral equation (2.43) when $\delta$ is small and the example from fracture mechanics. We shall show that the kernel $m_{1}(x, y)$, as defined by $(2.44)$, is $o(\delta)$. This implies that the rate of convergence of the Neumann series for this integral equation is $o\left(\delta^{2}\right)$. Hence

$$
u \sim F_{0}(x)+o\left(\delta^{2}\right),
$$

where $F_{0}(x)$ is defined by (2.47). For the fracture mechanics example we give $F_{0}(x)$ and show that the order estimate in $(3.1)$ is actually $O\left(\delta^{3} \log \delta\right)$.

The kernel $m_{1}(x, y)$ only involves $\delta$ through the function

$$
g(x, t)=\int_{0}^{\infty} b(x+s) b(t+s) d s, \quad t \geq 1,0<x<1 .
$$

We show below that

$$
g(x, t) \sim \delta b_{1}(t) \phi(x)+o(\delta), \quad t \geq 1,
$$

where

$$
\phi(x)=1-B(x)=\int_{x}^{\infty} b(t) d t
$$


$b_{1}(x)$ is defined by (1.23), and $B(x)$ is given by (2.26). When this approximation is substituted into (2.44), the result is $m_{1}(x, y) \sim o(\delta)$. Thus, we have the approximation (3.1).

To establish (3.3), we obtain an alternate representation of $g(x, t)$. From (2.10) and $n(t)=n(-t)$, we have

$$
g(0, t)=n(t) .
$$

Integration of (2.32) with respect to $x$ from 0 to $x$ yields

$$
g(x, t)=n(t) \phi(x)+[n(t)-b(t)] B(x)-\int_{0}^{x} g_{t}\left(x_{1}, t\right) d z d x_{1},
$$

where we have used the relation $\phi(0)=\hat{b}(0)=1$, which follows from (3.4) and (1.17). To obtain an asymptotic expression for $n(x), x>0$, we note that

$$
\begin{aligned}
n(x)= & \frac{1}{2 \pi} \int_{-\infty}^{\infty} e^{-i \xi x} \hat{n}(\xi) d \xi=\frac{1}{2 \pi} \int_{C_{\xi}} e^{-i \xi x} \hat{n}(\xi) d \xi \\
& \sim \frac{1}{2 \pi} \int_{C_{\xi}} e^{-i \xi x}\left\{c_{0}-\delta\left(\xi^{2}-\alpha^{2}\right) \hat{m}(\xi)+O\left(\delta^{2}\right)\right\} d \xi \sim \delta b_{1}(x)+O\left(\delta^{2}\right),
\end{aligned}
$$

where $C_{\xi}$ is the contour defined by $(1.11), b_{1}(x)$ is given by (1.23), and the relations (1.13) and (1.14) have been used. Thus, by (1.22) and (3.7), we have

$$
n(t)-b(t) \sim o(\delta), \quad t \geq 1 .
$$

By (3.2) and (1.22) we find that for $x$ near the boundary layer at $x=0$,

$$
g_{t}(x, t) \sim \int_{0}^{\infty} b(x+s) b_{1}^{\prime}(t+s) d s \sim O(\delta), \quad t \geq 1
$$

and that for $x$ outside the boundary layer,

$$
g_{t}(x, t) \sim \delta^{2} \int_{0}^{\infty} b_{1}(x+s) b_{1}^{\prime}(t+s) d s \sim O\left(\delta^{2}\right), \quad t \geq 1 .
$$

Thus

$$
\int_{0}^{x} g_{t}\left(x_{1}, t\right) d x_{1} \sim o(\delta) .
$$

Substitution of (3.7), (3.8), and (3.11) into (3.6) yields (3.3).

Thus we can make the approximation

$$
u(x) \sim F_{0}(x)+o\left(\delta^{2}\right),
$$

where $F_{0}(x)$ is given by (2.47). Also, if we substitute the approximation (3.3) into (2.47), we obtain the simpler approximation

$$
u(x) \sim \int_{0}^{x} \int_{0}^{x_{0}} b(s) b(y) f(x+y-s) d y d s+o(\delta) .
$$

If $f(x)$ is a polynomial or an exponential, the expression defining $F_{0}(x)$ simplifies. For example, if

$$
f(x)=1
$$


then

$$
\begin{aligned}
F_{0}(x)= & B(x) B\left(x_{0}\right)-[\phi(1)-\phi(x)]\left[\phi(1)-\phi\left(x_{0}\right)\right] \\
& +\left\{\int_{0}^{x}-\int_{x_{0}}^{1}\right\} g(1, y) d y-f_{1}+f_{1} W_{1}(x)+f_{2} W_{2}(x),
\end{aligned}
$$

where

$$
\begin{aligned}
& f_{1}=-\frac{1}{2} \phi^{2}(1)+\int_{1}^{\infty}[b(y)-n(y)] d y, \\
& f_{2}=\phi(1) b(1)-g(1,1)+n(1)-b(1) .
\end{aligned}
$$

Let us now consider the example from fracture mechanics: $m(x)=-\pi^{-1} \log |x|$ and $\alpha=0$. Then integral equation (1.1a) becomes

$$
u(x)+\frac{\delta}{\pi} \frac{d^{2}}{d x^{2}} \int_{0}^{1} \log |x-y| u(y) d y=f_{0}(x), \quad 0<x<1 .
$$

Since $\alpha=0, c_{0}=1$ and $f_{0}(x)=f(x)$. Also,

$$
\hat{n}(\xi)=1+\delta(-i \xi)^{1 / 2}(i \xi)^{1 / 2}
$$

where the branch of the square-root function $\sqrt{\xi}$ is the half line: $\operatorname{Re}\{\xi\}<0$ and $\operatorname{Im}\{\xi\}=0$. Then

$$
\begin{gathered}
n(x)=n(-x)=\frac{1}{\pi \delta} \int_{0}^{\infty} t\left(1+t^{2}\right)^{-1} e^{-x t / \delta} d t, \quad x>0, \\
b(x)=\frac{1}{\pi \delta} \int_{0}^{\infty} \frac{t}{\left(1+t^{2}\right)^{3 / 4}} \exp \left[-x t / \delta-\frac{1}{\pi} \int_{0}^{t} \frac{\log s}{1+s^{2}} d s\right] d t .
\end{gathered}
$$

From (3.20) and (3.21), we obtain the following asymptotic expressions:

$$
\begin{gathered}
n(x)=\delta b_{1}(x)+O\left(\delta^{3}\right), \quad x \gg \delta ; \\
b(x)=\delta b_{1}(x)+\delta^{2} b_{2}(x)+O\left(\delta^{3}\right), \quad x \gg \delta,
\end{gathered}
$$

where

$$
\begin{gathered}
b_{1}(x)=\left(\pi x^{2}\right)^{-1}=m^{\prime \prime}(x), \\
b_{2}(x)=\pi^{-2} x^{-3}\left[2 \gamma_{e}-1+2 \log (x / \delta)\right],
\end{gathered}
$$

and $\gamma_{e}=0.577 \ldots$ is Euler's constant. Note that (3.24) is consistent with (1.22). For this example, the approximation (3.3) becomes

$$
g(x, t) \sim \delta b_{1}(t)+O\left(\delta^{2} \log \delta\right) .
$$

Thus $m_{1}(x, t) \sim O\left(\delta^{2}-\log \delta\right)$ and the rate of convergence of the Neumann series for the integral equation $(2.43)$ is $O\left(\delta^{3} \log \delta\right)$. Hence we have the approximations

$$
\begin{gathered}
u \sim F_{0}(x)+O\left(\delta^{3} \log \delta\right), \\
u \sim \int_{0}^{x} \int_{0}^{x_{0}} b(s) b(y) f(x+y-s) d y d s+O\left(\delta^{2} \log \delta\right),
\end{gathered}
$$

where $F_{0}$ is given by (2.47). 
For the purpose of comparison, we offer explicit expansion of $u$ inside and outside the boundary layer for the case where $f_{0}(x)=1$. Using (3.15) to compute $F_{0}(x)$, we find that

$$
\begin{aligned}
u(x) \sim & \left\{1-\frac{\delta}{\pi}-\frac{2 \delta^{2}}{\pi^{2}}\left[\gamma_{e}-\log \delta-\frac{1}{4}\right]\right\} B(x) \\
& -\frac{\delta}{\pi} \int_{0}^{x}(x-y) b(y) d y+O\left(\delta^{3} \log \delta\right), \quad x=O(\delta) \\
u(x) \sim 1-\frac{\delta}{\pi x x_{0}}+ & \frac{\delta^{2}}{\pi^{2}}\left[\frac{2}{x x_{0}}-\left\{\gamma_{e}-\log \delta\right\}\left\{\frac{1}{x^{2}}+\frac{1}{x_{0}^{2}}\right\}\right. \\
+ & \left.\left\{\frac{1}{x_{0}^{2}}-\frac{1}{x^{2}}\right\} \log \left(x / x_{0}\right)\right]+O\left(\delta^{3} \log \delta\right), \quad x x_{0} \gg \delta .
\end{aligned}
$$

To compare our result with that of Willis and Nemat-Nasser [5] we need to introduce the function

$$
v(x)=\int_{0}^{x}(x-y) b(y) d y-x+\frac{\delta}{\pi}\left(\gamma_{e}+1\right) B(x) .
$$

It is easily shown that $\lim _{x \rightarrow \infty}\left[v(x)+\frac{\delta}{\pi} \log \frac{x}{\delta}\right]=0$ and

$$
\delta \frac{d^{2}}{d x^{2}} \int_{0}^{\infty} \log |x-y| v(y) d y+v(x)=-\frac{\delta}{\pi} \log \frac{x}{\delta}, \quad x>0 .
$$

This function is related to the function $V_{2}(x)$ defined by Willis and Nemat-Nasser [5] by some scalar factors. Our asymptotic expansions (3.29) and (3.30) are in agreement with those of Willis and Nemat-Nasser [5]. For this example, we also have

$$
u(x) \sim F_{0}(x)+\int_{0}^{1} m_{1}(x, y) B(y) B(1-y) d y+O\left(\delta^{5}(\log \delta)^{2}\right) .
$$

We can also consider the equation

$$
q(x) u(x)+\delta L_{x} L_{x}^{*} \int_{0}^{1} m(x-y) u(y) d y=f_{0}(x), \quad 0<x<1,
$$

with the conditions $u(0)=u(1)=0$, where $q(x)$ has the properties that $q(x)>0$, $0 \leq x \leq 1, q(x)=q(1-x)$, and $q(0)=1$. We define

$$
\tilde{f}_{0}(x)=f_{0}+(1-q(x)) u(x) .
$$

Then (3.34) becomes

$$
u(x)+\delta L_{x} L_{x}^{*} \int_{0}^{1} m(x-y) u(t) d y=\tilde{f}_{0}(x) .
$$

We can now use the result (2.43) to obtain

$$
q(x) u(x)+\delta \int_{0}^{1} m_{1}(x, y) u(y) d y+M_{1} u+M_{2} u=F_{0}(x), \quad 0<x<1,
$$


where $m_{1}$ and $F_{0}$ are given by (2.44) and (2.47), and

$$
\begin{aligned}
c_{0} M_{1} u= & -\int_{0}^{x} \int_{0}^{x_{0}} b(s) b(y)[1-q(x+y-s)] u(x+y-s) d y d s \\
& +c_{0}[1-q(x)] u(x) \\
c_{0} M_{2} u= & \int_{x_{0}}^{1} g(x, y)[1-q(y)] u(y) d y+\int_{0}^{x} g\left(x_{0}, 1-y\right)[1-q(y)] u(y) d y \\
& -\int_{0}^{1} g(1, y)[1-q(y)] u(y) d y W_{1}(x) \\
& -\int_{0}^{1} g_{x}(1, y)[1-q(y)] u(y) d y W_{2}(x) .
\end{aligned}
$$

As $\delta \rightarrow 0$, it can be shown that $M_{1} u \sim o(1)$ and $M_{2} u \sim o(\delta)$. Thus the Neumann series for (3.37) converges. Then we immediately have the approximation

$$
q(x) u(x) \sim \int_{0}^{x} \int_{0}^{x_{0}} b(s) b(y) f(x+y-s) d y d s+o(1) .
$$

For the fracture mechanics example $\left(\alpha=0, m(x)=-\frac{1}{\pi} \log |x|\right.$, and $\left.f_{0}(x)=1\right)$, we can write

$$
u(x) \sim u_{1}(x)+u_{2}(x)+O\left(\delta^{2}(\log \delta)^{2}\right),
$$

where

$$
\begin{gathered}
q(x) u_{1}(x)=B(x) B\left(x_{0}\right), \\
q(x) u_{2}(x)=[q(x)-1] u_{1}(x) \\
+\int_{0}^{x} \int_{0}^{x_{0}} b(s) b(y)[1-q(x+y-s)] u_{1}(x+y-s) d y d s .
\end{gathered}
$$

Acknowledgment. This research was carried out while the author was visiting the Center of Excellence for Advanced Materials at the University of California, San Diego.

This work was supported in part by the Applied Mathematical Sciences subprogram of the Office of Energy Research, U. S. Department of Energy under contract W-7405-ENG-82.

\section{REFERENCES}

[1] A. K. Gautesen, On the asymptotic solution to a class of linear integral equations, SIAM J. Appl. Math. 48, 294-306 (1988)

[2] M. Hori and S. Nemat-Nasser, Asymptotic solution to a class of strongly singular integral equations, SIAM J. Appl. Math. 50, 716-725 (1990)

[3] S. Nemat-Nasser and M. Hori, Toughening by partial or full bridging of cracks in ceramics and fiber reinforced composites, Mechics of Materials 6, 245-269 (1987)

[4] W. E. Olmstead and A. K. Gautesen, Asymptotic solution of some singularly perturbed Fredholm integral equations, Z. Angew. Math. Phys. 40, 230-244 (1989)

[5] J. R. Willis and S. Nemat-Nasser, Singular perturbation solution of a class of singular integral equations, Quart. Appl. Math. 48, 741-753 (1990) 